\title{
Quality of Field Reconstruction in Sensor Networks
}

\author{
A. Nordio, C.-F. Chiasserini, E. Viterbo \\ Dipartimento di Elettronica, Politecnico di Torino, Italy \\ Email: <name>@polito.it
}

\begin{abstract}
We consider the problem of obtaining a high quality estimates of band-limited sensor fields when sensor measurements are noisy and the nodes are irregularly deployed and subject to random motion. We consider the mean square error (MSE) of the estimate and we analytically derive the performance of several reconstruction/estimation techniques based on linear filtering. For each technique, we obtain the mean value of the MSE, as well as its asymptotic expression in the case where the field bandwidth and the number of sensors grow to infinity, while their ratio is kept constant. Our results provide useful guidelines for the design of sensor networks when many system parameters have to be traded off.
\end{abstract}

\section{INTRODUCTION}

Advances in wireless technology have enabled the use of wireless sensor networks for several important services, such as environmental and traffic control, habitat monitoring, and weather forecasts. Most of these applications require to sample a physical phenomenon over the network area, process the observed samples and communicate the resulting information to the external world.

In this paper, we consider a set of sensors communicating with a sink node, through either single- or multi-hop communications. While each sensor locally samples the physical field, the sink collecting all samples is in charge of reconstructing the signal of interest. We assume that initially sensors are either located at pre-defined positions, or, if randomly deployed over the network area, their location can be estimated at the sink node. We consider a fixed time instant and focus on the spatial sampling and reconstruction of the sensor field. We note that, in general, sensors provide an irregular sampling of the observed phenomenon. This may be due to various reasons: random deployment of the nodes, environment characteristics that bias the network deployment, sensors entering a sleep mode, inaccuracy in sensor positioning, or nodes movement [1]. In general the sink has to reconstruct the field from a collection of samples that are not equally spaced.

The problem of signal reconstruction from irregularly spaced samples has been widely addressed in signal processing. Several efficient and fast algorithms have been proposed to numerically reconstruct or approximate a signal [2], [3]. The problem we address in this work, however, is different. The questions we pose are as follows:

(i) how do noisy measures and uncertainty on the knowledge of the sensor positions affect the quality of the reconstructed signal?

(ii) how can we trade off system parameters like measurement noise level, field bandwidth, signal reconstruction quality and number of sensors?
The model of the monitoring system we analyze here assumes that the sensors position varies around an average value, and only the average location of the nodes is known at the sink. Examples where this model applies are observation systems using surface buoys [4], underwater robots located at different depths [5], dropsondes or low-cost unmanned platforms [6].

Our main results are as follows. We use as field reconstruction techniques some linear filters that are commonly employed in signal detection and estimation, and we evaluate the mean square error (MSE) of the obtained estimate. We find that a key parameter for the network performance is the ratio $\beta$ of the field bandwidth to the number of sampling sensors. In particular, there exists a critical value of this ratio beyond which the performance of all considered reconstruction strategies degrade significantly, even for low values of noise level and limited uncertainty on the sensor positions. To obtain an acceptable reconstruction quality when $\beta$ is large, reconstruction techniques that exploit some knowledge of the measurement noise and the sensor movement must be employed. We carry out an asymptotic analysis of the system as the field bandwidth and the number of sensors grow to infinity, while their ratio $\beta$ is kept constant, and we show that this is an effective tool to study the system performance even when the number of sensors is small. Finally, we find a lower bound to the MSE that can be achieved by any of the considered techniques.

\section{SYSTEM MODEL AND DIFFERENCES WITH RESPECT TO PREVIOUS WORK}

\section{A. Assumptions}

Let us consider a field with one-sided bandwidth $M$, sampled by $r$ sensors placed at positions $\mathbf{x}=\left[x_{1}, \ldots, x_{r}\right]^{\mathrm{T}}$, $x_{q} \in[0,1), q=1, \ldots, r$, which are in general not equally spaced.

We define $\beta$ as the ratio of the double-sided bandwidth of the field to the number of sensors, i.e., $\beta=(2 M+1) / r$. This is an important parameter in our analysis; note that, if a Nyquist sampling interval were used, we would have: $\beta=1$.

The field discrete spectrum is denoted by the $2 M+1$ column vector $^{1} \mathbf{a}=\left\{a_{k}\right\}, k=-M, \ldots, M$; we model the spectrum a as a random vector with covariance matrix $\mathbb{E}\left[\mathbf{a a}^{\dagger}\right]=\sigma_{a}^{2} \mathbf{I}_{2 M+1}$. This is a conservative assumption because

\footnotetext{
${ }^{1}$ Column vectors are denoted by bold lowercase letters, matrices are denoted by bold upper case letters. The $(k, q)$ entry of the matrix $\mathbf{X}$ is denoted by $(\mathbf{X})_{k q}$. The $n \times n$ identity matrix is denoted by $\mathbf{I}_{n}$, the generic identity matrix is denoted by $\mathbf{I}$, and the conjugate transpose operator is denoted by $(\cdot)^{\dagger}$
} 
it corresponds to the case where no a priori information is available on the observed signal. The value $\mathbf{s}$ of the field at positions $\mathrm{x}$ depends on the spectrum a through the expression $\mathbf{s}=\mathbf{G}_{\mathbf{x}}^{\dagger} \mathbf{a}$, where $\mathbf{s}$ is a size $r$ column vector and $\mathbf{G}_{\mathbf{x}}$ is the $(2 M+1) \times r$ generalized Fourier matrix defined as:

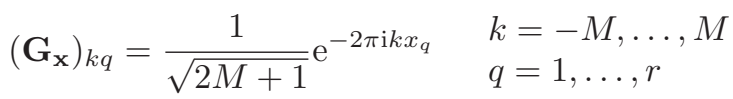

The dependence of the matrix $\mathbf{G}_{\mathbf{x}}$ on the position vector $\mathbf{x}$ is clearly indicated by its subscript. When the samples are equally spaced in the interval $[0,1), \mathbf{G}_{\mathbf{X}} \mathbf{G}_{\mathbf{x}}^{\dagger}$ is proportional to the identity matrix (i.e., $\mathbf{G}_{\mathbf{x}} \mathbf{G}_{\mathbf{x}}^{\dagger}=\mathbf{I} / \beta$ ).

Finally, we assume that sensor field measures are sent to a processing unit, the so-called sink node, whose task is to provide an estimate $\hat{\mathbf{a}}$ of the field spectrum a. Since in our work we focus on the reconstruction of the physical field, we consider that sensor transmissions always reach successfully the sink node.

\section{B. System model}

Here the characteristics of the model under study are summarized. Sensors move around the average position $\hat{\mathbf{x}}=\mathbb{E}[\mathbf{x}]$, i.e., the sensor positions are given by: $\mathrm{x}=\hat{\mathrm{x}}+\delta$, where $\delta$ is the relative position with respect to the average location $\hat{\mathbf{x}}$. The noisy measures $\mathbf{p}$ are given by:

$$
\mathbf{p}=\mathbf{s}+\mathbf{m}=\mathbf{G}_{\mathbf{x}}^{\dagger} \mathbf{a}+\mathbf{m}=\mathbf{G}_{\hat{\mathbf{x}}+\delta}^{\dagger} \mathbf{a}+\mathbf{m}
$$

The noise $\mathbf{m}$ and the relative positions $\delta$ are modeled as Gaussian random variables with $\mathbb{E}\left[\mathbf{m m}^{\dagger}\right]=\sigma_{m}^{2} \mathbf{I}_{r}$ and $\mathbb{E}\left[\boldsymbol{\delta} \boldsymbol{\delta}^{\dagger}\right]=\sigma_{\delta}^{2} \mathbf{I}_{r}$, respectively. The noise, the relative positions, and the field spectrum are considered mutually independent, i.e., $\mathbb{E}\left[\mathbf{m} \boldsymbol{\delta}^{\dagger}\right]=\mathbb{E}\left[\mathbf{m} \mathbf{a}^{\dagger}\right]=\mathbb{E}\left[\mathbf{a} \boldsymbol{\delta}^{\dagger}\right]=\mathbf{0}$. The sink has perfect knowledge of $\hat{\mathbf{x}}$.

\section{Our contribution with respect to previous work}

Not many papers have addressed the problem of sampling and reconstruction in sensor networks. Here we briefly review some of these works.

Given a network where sensors can enter a low-power operational state (i.e., a sleep mode), the work in [7] presents an algorithm to determine which sensor subsets should be selected to acquire data from an area of interest and which nodes should remain inactive to save energy. A similar problem is addressed in [8], where an adaptive sampling is described, which allows the central data-collector to vary the number of active sensors, i.e., samples, according to the desired resolution level. Note that, in our work, we consider an irregular topology that may be caused by nodes moving into a sleep state; however, we do not directly address energy efficiency and scheduling of the node sleep/activity periods.

In [9], the authors consider a unidimensional field, uniformly sampled at the Nyquist frequency by low-precision sensors. The impact on the field reconstruction accuracy of quantization errors and node density is evaluated. In our work we consider an additive noise that models errors due to the measurement procedure as well as to quantization of the samples, but we do not specifically focus on the latter issue.

The field reconstruction at the sink node with spatial and temporal correlation among sensor measures is studied in [10]-[13]. The problem of reconstructing a band-limited signal from an irregular set of samples at unknown locations is addressed in [14]. There, sensor positions are unknown but always equal to an integer multiple of the sampling interval. Differently from [14], we assume that the sink can either acquire or estimate the sensor locations and that sensors are randomly deployed over a subset of $\mathbb{R}$. Finally, in our previous work [15] some conditions on the irregular topology of the sensor network are identified, which allow for a successful signal reconstruction, both under deterministic and random node deployment. Here, we propose to apply linear filters to the field reconstruction and we consider the following causes of quality degradation: (i) noisy measures and (ii) uncertainty at the sink on the position of moving sensors.

\section{PRELIMINARIES}

Several reconstruction techniques have been proposed in the literature, which amount to the solution of a linear system (see [2], [3] and the references cited therein). A widely used technique consists in processing the measures $\mathbf{p}$ by means of a linear filter, $\mathbf{B}$, which is an $r \times(2 M+1)$ matrix and is a function of the system parameters known at the sink. Then the estimate of the field spectrum is given by:

$$
\hat{\mathbf{a}}=\mathbf{B}^{\dagger} \mathbf{p}
$$

\section{A. Our performance metrics}

Given the spectrum estimates $\hat{\mathbf{a}}$, for each location $x$ the field estimate $\hat{s}(x)$ can be obtained as: $\hat{s}(x)=$ $\sum_{k=-M}^{M} \exp (\mathrm{j} 2 \pi k x) \hat{a}_{k} / \sqrt{2 M+1}$. Therefore, a high quality field reconstruction is possible if a very precise estimate $\hat{a}$ is provided. As a measure of the estimate precision of the field spectrum, given the average locations $\hat{\mathbf{x}}$ we employ the mean square error (MSE) defined as ${ }^{2}$

$$
\mathrm{MSE}_{\hat{\mathbf{x}}}=\underset{\mathbf{a}, \mathbf{m}, \delta}{\mathbb{E}}\left[\|\hat{\mathbf{a}}-\mathbf{a}\|^{2}\right]=\sigma_{a}^{2} \operatorname{Tr}\left\{\boldsymbol{\Phi}_{\hat{\mathbf{x}}}\right\}
$$

where $\boldsymbol{\Phi}_{\hat{\mathbf{x}}} \triangleq \frac{1}{\sigma_{a}^{2}} \underset{\mathbf{a}, \mathbf{m}, \delta}{\mathbb{E}}\left[(\hat{\mathbf{a}}-\mathbf{a})(\hat{\mathbf{a}}-\mathbf{a})^{\dagger}\right]$. Using (3), after some algebra we have:

$$
\mathbf{\Phi}_{\hat{\mathbf{x}}}=\mathbf{B}^{\dagger}\left(\underset{\delta}{\underset{\delta}{\mathbb{E}}}\left[\mathbf{G}_{\mathbf{x}}^{\dagger} \mathbf{G}_{\mathbf{x}}\right]+\alpha \mathbf{I}\right) \mathbf{B}-2 \Re\left\{\underset{\delta}{\mathbb{E}}\left[\mathbf{G}_{\mathbf{x}}\right] \mathbf{B}\right\}+\mathbf{I}
$$

where $\mathrm{SNR}_{m}=1 / \alpha=\sigma_{a}^{2} / \sigma_{m}^{2}$ is the signal-to-noise ratio on the measure. Next, we consider the vector $\hat{\mathrm{x}}$ to be random with entries uniformly distributed over the interval $[0,1)$. In this case a more appropriate performance metric is the per-sample average MSE, normalized to $\sigma_{a}^{2}$, i.e., $\mathrm{MSE}_{\mathrm{av}}=$ $\mathbb{E}\left[\mathrm{MSE}_{\hat{\mathbf{x}}}\right] /(2 M+1) / \sigma_{a}^{2}$ where $\mathrm{MSE}_{\hat{\mathbf{x}}}$ is as in (4). When the parameters $M$ and $r$ grow to infinity while the ratio

\footnotetext{
${ }^{2}$ The operator $\underset{\mathrm{E}}{\mathbb{E}}[\cdot]$ averages the argument over the distribution of the random vector $\mathbf{v}, \operatorname{v} \operatorname{Tr}\{\cdot\}$ is the trace operator
} 
$\beta=(2 M+1) / r$ is kept constant, we also consider the asymptotic per-sample average MSE, defined as:

$$
\mathrm{MSE}_{\infty}=\lim _{\substack{M, r \rightarrow+\infty \\ \frac{2 M+1}{r}=\beta}} \mathrm{MSE}_{\mathrm{av}}
$$

\section{B. Some useful mathematical tools}

1) The functional $\phi$ : Let us first consider an $n \times n$ Hermitian random matrix $\mathbf{X}$ and the functional $\phi(\mathbf{X}) \triangleq$ $\lim _{n \rightarrow+\infty} \frac{1}{n} \mathbb{E}[\operatorname{Tr}\{\mathbf{X}\}]$. In our analysis we use the following results on the functional $\phi(\cdot)$ [16]. First, we notice that $\phi(\mathbf{I})=1$. Secondly, if $g(x)$ is a continuous function defined in $x \in \mathbb{R}^{+}$with existing Taylor expansion in $x \in \mathbb{R}^{+}$, then

$$
\phi(g(\mathbf{X}))=\mathbb{E}[g(\xi)]
$$

where $\xi$ is the random variable distributed as the asymptotic eigenvalues of $\mathbf{X}$.

Using (6), the asymptotical MSE can be written as:

$$
\mathrm{MSE}_{\infty}=\phi\left(\boldsymbol{\Phi}_{\hat{\mathbf{x}}}\right)
$$

2) A simple expression for $\mathbf{G}_{\hat{\mathbf{x}}+\delta}$ : As will be clear in Sec. IV, many parameters are functions of the matrix $\mathbf{G}_{\hat{\mathbf{x}}+\delta}$. It is thus useful to derive an expression of $\mathbf{G}_{\mathbf{x}}$ as a function of $\mathbf{G}_{\hat{\mathbf{x}}}$, in order to separate the random part, $\delta$, of $\mathbf{x}$ from the constant part $\hat{\mathbf{x}}$. From (1), the $(k, q)$ entry of $\mathbf{G}_{\mathbf{x}}$ is defined as:

$$
\left(\mathbf{G}_{\mathbf{x}}\right)_{k q}=\frac{\mathrm{e}^{-2 \pi \mathrm{i} k x_{q}}}{\sqrt{2 M+1}}=\frac{\mathrm{e}^{-2 \pi \mathrm{i} k \hat{x}_{q}} \mathrm{e}^{-2 \pi \mathrm{i} k \delta_{q}}}{\sqrt{2 M+1}}
$$

A useful expression of $\mathbf{G}_{\hat{\mathbf{x}}}$ in terms of $\mathbf{G}_{\mathbf{x}}$ is given by the following theorem.

Theorem III.1 For any vector $\mathbf{x}$ of size $r$, let the $(k, q)$ entry of the matrix $\mathbf{G}_{\mathbf{x}}$ be $\left(\mathbf{G}_{\mathbf{x}}\right)_{k q}=\exp \left(-2 \pi \mathrm{i} k x_{q}\right) / \sqrt{2 M+1}$ for $k=-M, \ldots, M$, and $q=1, \ldots, r$. Let the size $r$ column vectors $\mathrm{x}, \hat{\mathrm{x}}$, and $\boldsymbol{\delta}$ be such that $\mathrm{x}=\hat{\mathrm{x}}+\boldsymbol{\delta}$ then

$$
\mathbf{G}_{\mathbf{x}}=\sum_{n=0}^{\infty} \frac{1}{n !} \mathbf{W}^{n} \mathbf{G}_{\hat{\mathbf{x}}} \Delta^{n}
$$

where $\boldsymbol{\Delta}=\operatorname{diag}(\boldsymbol{\delta})$ is an $r \times r$ diagonal matrix, and $\mathbf{W}$ is a $(2 M+1) \times(2 M+1)$ diagonal matrix with $(\mathbf{W})_{k k}=-2 \pi \mathrm{i} k$.

Proof: Omitted; it can be found in [17], available on line.

\section{Model Analysis}

As already mentioned in Section II, here we consider the case where sensors are moving and only their average position, $\hat{\mathbf{x}}$, is known at the sink node. The reconstruction algorithm employs the matrix $\mathbf{B}$, which is a function of the known average positions $\hat{\mathbf{x}}$. For any given average location $\hat{\mathbf{x}}$ and filter matrix $\mathrm{B}$, the MSE of the reconstruction is given by (4) and (5). To proceed further, we need to compute the averages (5) over the relative positions $\delta$, i.e., we need the expression of $\underset{\delta}{\mathbb{E}}\left[\mathbf{G}_{\mathbf{x}}\right]$ and $\underset{\delta}{\mathbb{E}}\left[\mathbf{G}_{\mathbf{x}}^{\dagger} \mathbf{G}_{\mathbf{x}}\right]$ as functions of $\mathbf{G}_{\hat{\mathbf{x}}}$. Through some simple calculations and exploiting Theorem III.1, we obtain [17]:

$$
\underset{\delta}{\mathbb{E}}\left[\mathbf{G}_{\mathbf{x}}\right]=\mathbf{M G}_{\hat{\mathbf{x}}}
$$

where $\mathbf{M}=\exp \left(\sigma_{\delta}^{2} \mathbf{W}^{2} / 2\right)$ is a $(2 M+1) \times(2 M+1)$ diagonal matrix with $(\mathbf{M})_{k k}=\exp \left(-2 \pi^{2} k^{2} \sigma_{\delta}^{2}\right), k=-M, \ldots, M$, and

$$
\underset{\boldsymbol{\delta}}{\mathbb{E}}\left[\mathbf{G}_{\mathbf{x}}^{\dagger} \mathbf{G}_{\mathbf{x}}\right]=\left[\mathbf{G}_{\hat{\mathbf{x}}}^{\dagger} \mathbf{M}^{2} \mathbf{G}_{\hat{\mathbf{x}}}+\left(1-\frac{\operatorname{Tr}\left\{\mathbf{M}^{2}\right\}}{2 M+1}\right) \mathbf{I}\right]
$$

The above definition of $\mathbf{M}$ is derived under the assumption that $\delta$ has a zero mean Gaussian distribution. However, we observe that the matrix $\mathbf{M}$, which depends on the distribution of the entries of $\delta$, can be written in a more general manner as: $(\mathbf{M})_{k k}=M_{\delta}(-2 \pi \mathrm{i} k), k=-M, \ldots, M$, where $M_{\delta}(\cdot)$ is the characteristic function of the relative positions. By doing so, the results reported in this section hold for any distribution of the entries of $\boldsymbol{\delta}$.

Using (10) and (11) in (5), we obtain:

$$
\boldsymbol{\Phi}_{\hat{\mathbf{x}}}=\mathbf{B}^{\dagger}\left(\mathbf{G}_{\hat{\mathbf{x}}}^{\dagger} \mathbf{M}^{2} \mathbf{G}_{\hat{\mathbf{x}}}+\gamma \mathbf{I}\right) \mathbf{B}-2 \Re\left\{\mathbf{M G}_{\hat{\hat{\mathbf{x}}}} \mathbf{B}\right\}+\mathbf{I}
$$

where $\gamma=1+\alpha-\frac{\operatorname{Tr}\left\{\mathbf{M}^{2}\right\}}{2 M+1}$ and $\Re\{\cdot\}$ represents the real part of the argument. In the following, we first consider that the variance $\sigma_{\delta}^{2}$ of the sensor movement is unknown at the sink and, hence, the sink assumes the sensors to be fixed (i.e., $\delta=$ 0 ). Then, we consider that $\sigma_{\delta}^{2}$ is known and the reconstruction algorithm employs a filter that exploits such an information to minimize the MSE.

\section{A. ZF filter}

In this case the sink node employs the $\mathrm{ZF}$ filter defined as $\mathbf{B}^{\dagger}=\mathbf{R}_{\hat{\mathbf{x}}}^{-1} \mathbf{G}_{\hat{\mathbf{x}}}$ [18], where $\mathbf{R}_{\hat{\mathbf{x}}}=\mathbf{G}_{\hat{\mathbf{x}}} \mathbf{G}_{\hat{\mathbf{x}}}^{\dagger}$. Notice that the sink knows only the average value of the sensor positions, therefore B results to be a function of $\hat{\mathbf{x}}$. Using (12), the matrix $\boldsymbol{\Phi}_{\hat{\mathbf{x}}}$ can be written as:

$$
\boldsymbol{\Phi}_{\hat{\mathbf{x}}}=\gamma \mathbf{R}_{\hat{\mathbf{x}}}^{-1}+(\mathbf{M}-\mathbf{I})^{2}
$$

We are now interested in deriving the asymptotic expression for the MSE. Note that the $\boldsymbol{\Phi}_{\hat{\mathbf{x}}}$ is a function of $\mathbf{R}_{\hat{\mathbf{x}}}$ and $\mathbf{M}$, and it contains terms of the form $g\left(\mathbf{R}_{\hat{\mathbf{x}}}\right)$ with $g(x)=x^{-1}$ and $\mathbf{M}^{p}$ with $p=1,2$. Also, the matrix $\mathbf{R}_{\hat{\mathbf{x}}}$ depends on $M$ and $r$, while the matrix $\mathbf{M}$ depends on $M$ and $\sigma_{\delta}^{2}$. The definition of $\mathrm{MSE}_{\infty}$ in (6) refers to the case where the bandwidth $M$ and the number of sensors $r$ grow to infinity with constant ratio $\beta$. If this definition is directly applied, information losses may arise; indeed, we have:

$$
\phi\left(\mathbf{M}^{p}\right)=\lim _{M \rightarrow+\infty} \frac{1}{2 M+1} \sum_{k=-M}^{M} \mathrm{e}^{-2 p \pi^{2} k^{2} \sigma_{\delta}^{2}}=0
$$

and thus all terms depending on the matrix $\mathbf{M}$ would vanish regardless of the value of $\sigma_{\delta}^{2}$. On the contrary, in a realistic situation we expect to obtain a high reconstruction quality when the standard deviation of the motion $\left(\sigma_{\delta}\right)$ is smaller than or comparable to the average sensor separation $(1 / r)$, and a significant degradation of the reconstruction quality when $\sigma_{\delta}$ is much larger than the average sensor separation. To distinguish such different conditions, we define the signal-to-noise ratio on the motion as: $\operatorname{SNR}_{x}=(1 / r)^{2} / \sigma_{\delta}^{2}=1 / \omega^{2}$ where $\omega=$ $\sigma_{\delta} r$. We then redefine the asymptotic MSE as the limit of the 
average MSE for $M, r \rightarrow+\infty$, with constant $\beta=(2 M+1) / r$ and constant $\omega=\sigma_{\delta} r$. In this case,

$$
\begin{gathered}
\phi\left(\mathbf{M}^{p}\right)=\lim _{\substack { M, r \rightarrow+\infty \\
\begin{subarray}{c}{\delta \\
\beta, \omega{ M , r \rightarrow + \infty \\
\begin{subarray} { c } { \delta \\
\beta , \omega } }\end{subarray}} \frac{1}{2 M+1} \sum_{k=-M}^{M} \exp \left(-2 p \pi^{2} k^{2} \sigma_{\delta}^{2}\right) \\
=\int_{-1 / 2}^{1 / 2} \exp \left(-2 p \pi^{2} z^{2} \beta^{2} \omega^{2}\right) \mathrm{d} z=\nu\left(\sqrt{\frac{p}{2}} \beta \omega\right)
\end{gathered}
$$

where $\nu(x)=\sqrt{\pi / 4} \operatorname{erf}(\pi x) /(\pi x)$. Notice that $\nu(0)=1$ and $\lim _{x \rightarrow+\infty} \nu(x)=0$. Also, we have:

$$
\phi(\gamma)=1+\alpha-\lim _{\substack{M, r \rightarrow+\infty \\ \sigma_{\delta} \rightarrow 0 \\ \beta, \omega}} \frac{\operatorname{Tr}\left\{\mathbf{M}^{2}\right\}}{2 M+1}=1+\alpha-\nu(\beta \omega)
$$

Using the new definition, the asymptotic expression of the MSE becomes:

$$
\begin{gathered}
\operatorname{MSE}_{\infty}=\phi\left(\boldsymbol{\Phi}_{\hat{\mathbf{x}}}\right)=\phi\left(\gamma \mathbf{R}_{\hat{\mathbf{x}}}^{-1}+(\mathbf{M}-\mathbf{I})^{2}\right) \\
=1+\beta(1+\alpha-\nu(\beta \omega)) \mathbb{E}\left[\frac{1}{\lambda}\right]+\nu(\beta \omega)-2 \nu\left(\frac{\beta \omega}{\sqrt{2}}\right)
\end{gathered}
$$

where $\lambda$ is the random variable distributed as the asymptotic eigenvalues of $\beta \mathbf{R}_{\hat{\mathbf{x}}}$ [15].

\section{B. MMSE filter neglecting $\sigma_{\delta}^{2}$}

Similarly, if the sink employs the filter $\mathbf{B}$ defined as $\mathbf{B}^{\dagger}=$ $\mathbf{A}^{-1} \mathbf{G}_{\hat{\mathbf{x}}}$ [18], with $\mathbf{A}=\mathbf{R}_{\hat{\mathbf{x}}}+\alpha \mathbf{I}$, then $\boldsymbol{\Phi}_{\hat{\mathbf{x}}}$ in (12) becomes:

$$
\boldsymbol{\Phi}_{\hat{\mathbf{x}}}=\mathbf{M}^{2} \mathbf{R}_{\hat{\mathbf{x}}} \mathbf{A}^{-2} \mathbf{R}_{\hat{\mathbf{x}}}+\gamma \mathbf{R}_{\hat{\mathbf{x}}} \mathbf{A}^{-2}-2 \Re\left\{\mathbf{M R}_{\hat{\mathbf{x}}} \mathbf{A}^{-1}\right\}+\mathbf{I}
$$

If $\mathbf{M}$ and $\mathbf{R}_{\hat{\mathbf{x}}}$ are asymptotically free matrices [16], then $\phi\left(\mathbf{M}^{p} \mathbf{R}_{\hat{\mathbf{x}}}^{q}\right)=\phi\left(\mathbf{M}^{p}\right) \phi\left(\mathbf{R}_{\hat{\mathbf{x}}}^{q}\right)$ for any positive integer $p$ and $q$. Hence, after some calculations and using (14), we obtain:

$$
\begin{gathered}
\operatorname{MSE}_{\infty}=1+(\nu(\beta \omega)-2 \nu(\beta \omega / \sqrt{2})) \mathbb{E}\left[\frac{\lambda^{2}}{(\lambda+\alpha \beta)^{2}}\right] \\
+\beta(1+\alpha-\nu(\beta \omega)-2 \alpha \nu(\beta \omega / \sqrt{2})) \mathbb{E}\left[\frac{\lambda}{(\lambda+\alpha \beta)^{2}}\right]
\end{gathered}
$$

Here we employed the property (7) with $g(x)=x^{2} /(x+\alpha)^{2}$, $g(x)=x /(x+\alpha)^{2}$, and $g(x)=x /(x+\alpha)$, respectively. Moreover since $\mathbf{R}_{\hat{\mathbf{x}}} \mathbf{A}^{-1}$ is Hermitian and $\mathbf{M}$ is real and diagonal $\operatorname{Tr}\left\{\Re\left\{\mathbf{M R}_{\hat{\mathbf{x}}} \mathbf{A}^{-1}\right\}\right\}=\operatorname{Tr}\left\{\mathbf{M} \mathbf{R}_{\hat{\mathbf{x}}} \mathbf{A}^{-1}\right\}$.

\section{MMSE filter for known $\sigma_{\delta}^{2}$}

We now consider the linear MMSE filter optimized for the case where $\sigma_{\delta}^{2}$ is known at the sink. We find the optimal $\mathbf{B}$ minimizing (12); that is, we null the derivative of (4) with respect to $\mathbf{B}$. We employ the following properties that hold for any square Hermitian matrix $\mathbf{X}$ :

$$
\frac{\partial}{\partial \mathbf{B}} \Re \operatorname{Tr}\{\mathbf{X B}\}=\mathbf{X}^{\dagger} ; \quad \frac{\partial}{\partial \mathbf{B}} \operatorname{Tr}\left\{\mathbf{B}^{\dagger} \mathbf{X B}\right\}=2 \mathbf{X B}
$$

Then, we have:

$$
\frac{\partial \mathrm{MSE}_{\hat{\mathbf{x}}}(\mathbf{B})}{\partial \mathbf{B}}=2 \sigma_{a}^{2}\left(\mathbf{G}_{\hat{\mathbf{x}}}^{\dagger} \mathbf{M}^{2} \mathbf{G}_{\hat{\mathbf{x}}}+\gamma \mathbf{I}\right) \mathbf{B}-2 \sigma_{a}^{2} \mathbf{G}_{\hat{\mathbf{x}}}^{\dagger} \mathbf{M}=\mathbf{0}
$$

Solving for $\mathbf{B}$, we obtain the expression of the MMSE filter

$$
\mathbf{B}^{\dagger}=\left(\mathbf{M R}_{\hat{\mathbf{x}}} \mathbf{M}+\gamma \mathbf{I}\right)^{-1} \mathbf{M G}_{\hat{\mathbf{x}}}
$$



Fig. 1. Performance of the ZF filter for $\beta=0.2$, when $\sigma_{\delta}^{2}$ is neglected

Substituting (17) into (12), we have: $\boldsymbol{\Phi}_{\hat{\mathbf{x}}}=$ $\gamma\left(\mathbf{M R}_{\hat{\mathbf{x}}} \mathbf{M}+\gamma \mathbf{I}\right)^{-1}$. In this case an explicit expression of $\mathrm{MSE}_{\infty}$ is hard to obtain. However, we were able to find the following lower bound that turned out to be very tight, as shown by the results presented in Sec. IV-D. Since $\phi(\cdot)$ is a convex function and $\underset{\hat{\mathbf{x}}}{\mathbb{E}}\left[\mathbf{R}_{\hat{\mathbf{x}}}\right]=1 / \beta[15]$ we have:

$$
\begin{gathered}
\operatorname{MSE}_{\infty}=\phi\left(\gamma\left(\mathbf{M R}_{\hat{\mathbf{x}}} \mathbf{M}+\gamma \mathbf{I}\right)^{-1}\right) \\
\geq \frac{1}{\phi\left(\frac{1}{\gamma}\left(\mathbf{M R}_{\hat{\mathbf{x}}} \mathbf{M}+\gamma \mathbf{I}\right)\right)}=\frac{\beta(1+\alpha-\nu(\beta \omega))}{\beta(1+\alpha)+(1-\beta) \nu(\beta \omega)}
\end{gathered}
$$

\section{Results}

We now show the performance of the analyzed filters. With regards to the ZF filter, Fig. 1 compares the asymptotic MSE evaluated through (15) (represented by solid lines and labeled by "MSE ${ }_{\infty}$ ") against the average MSE (represented by points and labeled by "MSE $\mathrm{av}_{\mathrm{av}}$ "). The $\mathrm{MSE}_{\mathrm{av}}$ is obtained by generating 100 realizations of the measures as in (2), with $M=10$, computing the estimates as in (3) and averaging the square error $\|\mathbf{a}-\hat{\mathbf{a}}\|^{2}$. The MSE is shown in the log scale plotted versus $\mathrm{SNR}_{m}$, for $\beta=0.2$ and different values of $\mathrm{SNR}_{x}$. The solid line labeled "SNR$x=+\infty$ " corresponds to the case where the sink has perfect knowledge of the sensor positions $(\boldsymbol{\delta}=\mathbf{0})$. The excellent match between the asymptotic results and the numerical simulation, even for moderate values of $M$ end $r$ confirms the validity of the asymptotic analysis as an effective tool to characterize the performance of the reconstruction techniques.

Fig. 2 compares the performance of the MMSE filter (17), which has knowledge of $\sigma_{\delta}^{2}$, with its lower bound (18), as $\mathrm{SNR}_{m}$ varies. We consider $\beta=0.2$ and different values of $\mathrm{SNR}_{x}$. Notice that the lower bound is very tight, especially for high values of $\mathrm{SNR}_{x}$.

The advantage of knowing $\sigma_{\delta}^{2}$ becomes significant for high values of $\beta$. This is shown in Fig. 3, where the performance of the MMSE filter (17) labeled by "MMSE $\sigma_{\delta}^{2}$ " (dashed 


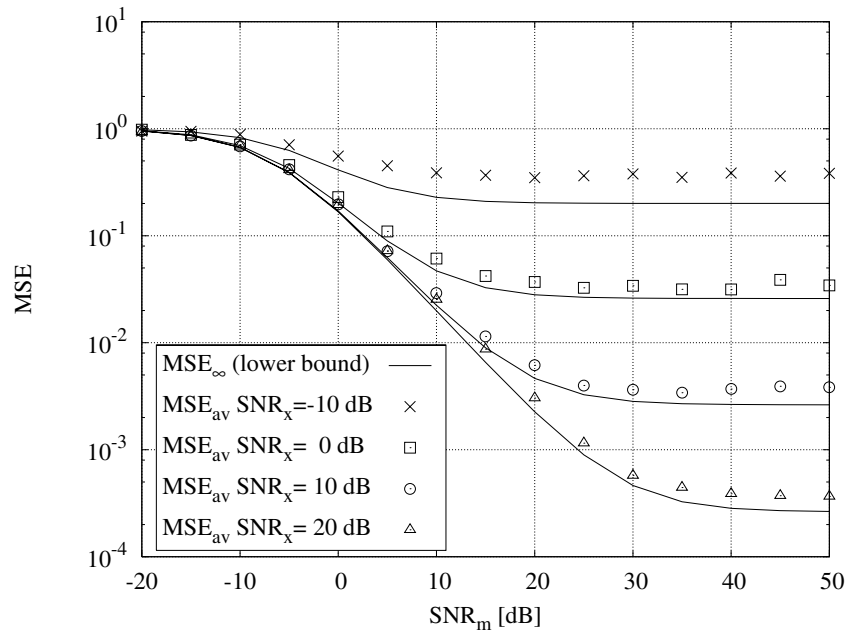

Fig. 2. Performance of the MMSE filter (17) with perfect knowledge of $\sigma_{\delta}^{2}$ versus $\mathrm{SNR}_{m}$, for $\beta=0.2$

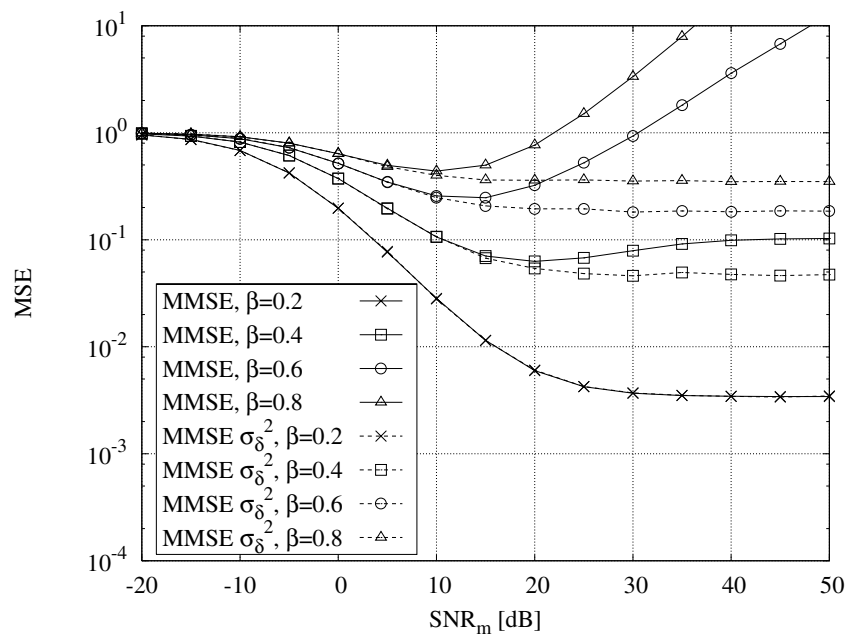

Fig. 3. Performance comparison of the MMSE filter neglecting $\sigma_{\delta}^{2}$ against the MMSE filter with perfect knowledge of $\sigma_{\delta}^{2}$, as $\mathrm{SNR}_{m}$ varies and for $\mathrm{SNR}_{x}=10 \mathrm{~dB}$

lines) and the performance of the MMSE filter neglecting $\sigma_{\delta}^{2}$, labeled by "MMSE" (solid lines), are compared for $\mathrm{SNR}_{x}=$ $10 \mathrm{~dB}$ and $\beta=0.2,0.4,0.6,0.8$. The two filters show similar performance only for $\beta=0.2$, while for larger $\beta$ 's the advantage provided by the knowledge of $\sigma_{\delta}^{2}$ becomes evident, especially for high values of $\mathrm{SNR}_{m}$. Indeed, the $\mathrm{MSE}_{\infty}$ of the MMSE filter neglecting $\sigma_{\delta}^{2}$ tends to 1 for small $\mathrm{SNR}_{m}$, but diverges as $\mathrm{SNR}_{m}$ increases, when $\beta>\beta^{\star} \approx 0.35$. This is because, for $\alpha \rightarrow 0$, the term $\left.\mathbb{E}\left[\lambda /(\lambda+\alpha \beta)^{2}\right)\right]$ in (16) tends to $\mathbb{E}[1 / \lambda]$ which diverges for $\beta>\beta^{\star}$ [15]. Furthermore, it is interesting to notice that the performance of the MMSE filter neglecting $\sigma_{\delta}^{2}$ shows a minimum which can be obtained by nulling the derivative of (16) with respect to $\alpha$.

\section{CONCLUSiOnS}

We addressed the problem of reconstructing band-limited fields from measurements taken by irregularly deployed sen- sors, and we studied effects of noisy measures and moving sensors on the reconstruction quality. We analytically derived the performance of several linear filters in terms of the MSE. We found that asymptotic analysis is an effective tool to characterize the performance of the reconstruction techniques even for a small number of sensors, and that the parameter $\beta$ has a key impact on the performance of our system. Our main results are: (i) the MSE provided by any of the reconstruction techniques is lower bounded by (18); (ii) the performance of all reconstruction techniques worsen with increasing $\beta$ and $\mathrm{SNR}_{x}$; (iii) the advantage of exploiting the knowledge of $\mathrm{SNR}_{x}$ in the filter design is of fundamental importance to obtain a high quality reconstruction for $\beta>0.35$ and large values of $\mathrm{SNR}_{m}$.

\section{REFERENCES}

[1] D. Ganesan, S. Ratnasamy, H. Wang, and D. Estrin, "Coping with irregular spatio-temporal sampling in sensor networks," in Proc. 2nd Workshop on Hot Topics in Networks, Cambridge, MA, Nov. 2003.

[2] H. G. Feichtinger, K. Gröchenig, and T. Strohmer, "Efficient numerical methods in non-uniform sampling theory," Numerische Mathematik, vol. 69 , pp. 423-440, 1995.

[3] K. Gröchenig, "Irregular sampling, toeplitz matrices, and the approximation of entire functions of exponential type," Mathematics of Computation, vol. 68, no. 226, pp. 749-765, Apr. 1999.

[4] "The autonomous ocean sampling network (aosn) project." [Online]. Available: http://www.mbari.org/aosn/

[5] E. Cayirci, H. Tezcan, Y. Dogan, and V. Coskun, "Wireless sensor networks for underwater surveillance systems," Ad Hoc Networks, vol. 4, no. 4, pp. 431-446, July 2006.

[6] S. J. Majumdar, S. D. Aberson, C. H. Bishop, R. Buizza, M. S. Peng, and C. A. Reynolds, "A comparison of adaptive observing guidance for atlantic tropical cyclones," in Proc. 27th Conference on Hurricanes and Tropical Meteorology, Apr. 2006.

[7] M. Perillo, Z. Ignjatovic, and W. Heinzelman, "An energy conservation method for wireless sensor networks employing a blue noise spatial sampling technique," in Proc. IPSN '04, Apr. 2004.

[8] R. Willett, A. Martin, and R. Nowak, "Backcasting: adaptive sampling for sensor networks," in Proc. IPSN '04, Apr. 2004

[9] P. Ishwar, A. Kumar, and K. Ramchandran, "Distributed sampling for dense sensor networks: a bit-conservation principle," in Proc. IPSN '03, Apr. 2003.

[10] R. Cristescu and M. Vetterli, "On the optimal density for real-time data gathering of spatio-temporal processes in sensor networks," in Proc. IPSN '05, Los Angeles, CA, Apr. 2005.

[11] Y. Sung, L. Tong, and H. Poor, "Sensor activation and scheduling for field detection in large sensor arrays," in Proc. IPSN '05, Los Angeles, CA, Apr. 2005

[12] M. C. Vuran, O. B. Akan, and I. F. Akyildiz, "Spatio-temporal correlation: theory and applications for wireless sensor networks," Computer Networks, vol. 45, no. 3, pp. 245-259, June 2004.

[13] Y. Rachlin, R. Negi, and P. Khosla, "Sensing capacity for discrete sensor network applications," in Proc. IPSN '05, Los Angeles, CA, Apr. 2005.

[14] P. Marziliano and M. Vetterli, "Reconstruction of irregularly sampled discrete-time bandlimited signals with unknown sampling locations," IEEE Trans. Sig. Processing, vol. 48, no. 12, pp. 3462-3471, Dec. 2000.

[15] A. Nordio, C.-F. Chiasserini, and E. Viterbo, "Bandlimited field reconstruction for wireless sensor networks," Politecnico di Torino, Tech. Rep., January 2006. [Online]. Available: http://www.telematica. polito.it/ nordio/sensors/TIT_sub.pdf

[16] A. Tulino and S. Verdú, "Random matrix theory and wireless communications," Foundations and Trends in Communications and Information Theory, vol. 1, no. 1, 2004.

[17] A. Nordio, C.-F. Chiasserini, and E. Viterbo, "Some useful mathematical tools," Politecnico di Torino, Tech. Rep., July 2006. [Online]. Available: http://www.telematica.polito.it/ nordio/sensors/TechRep.pdf

[18] S. Verdù, Multiuser detection. Cambridge, UK: Cambridge Univ. Press, 1998. 